\section{俩 Heighten Science \\ P U B L I C I T I O N S Corporation ISSN 2576-9537}

\title{
New era of liver transplantation for HIV-HCV Co-infected patients: A case report
}

\author{
Konstantinos A Zorbas*, Sunil S Karhadkar, Kwan N Lau, \\ Andreas Karachristos and Antonio Di Carlo \\ Lewis Katz School of Medicine at Temple University Hospital, Department of Surgery, Philadelphia, PA, USA
}

\begin{abstract}
*Address for Correspondence: Konstantinos A Zorbas, Lewis Katz School of Medicine at Temple University Hospital, $3401 \mathrm{~N}$ Broad St, Philadelphia, PA 19140, USA, Tel: 2672942932 Email: konstantinos.zorbas@tuhs.temple.edu
\end{abstract}

Submitted: 03 November 2017

Approved: 13 November 2017

Published: 14 November 2017

Copyright: @ 2017 Zorbas KA, et al. This is an open access article distributed under the Creative Commons Attribution License, which permits unrestricted use, distribution, and reproduction in any medium, provided the original work is properly cited

Keywords: Liver Transplantation; HIV; HCV; Immunosuppression; Direct Acting Antivirals; Ledipasvir/Sofosbuvir

Abbreviations: ESLD: End Stage Liver Disease; HIV: Human Immunodeficiency Virus; HCV: Hepatitis C Virus, HAART: Highly Active Antiretroviral Therapy; OLT: Orthotopic Liver Transplantation; DAAs: Direct-Acting Antivirus Regiment; HCC: Hepatocellular Carcinoma; SVR: Sustained Virologic Response; CNIs: Calcineurin Inhibitors

Research highlights: The advent of DAA drugs has given the opportunity in HCV-HIV population to counter the deleterious effect of $\mathrm{HCV}$ on liver allografts after transplantation. In our opinion HIV-HCV co-infection is no longer a contraindication for liver transplantation.

\section{Abstract}

Morbidity and mortality of HIV-infected patients have been improved over the last decades with the advent of combined antiretroviral therapy. As a result, other comorbidities such as chronic kidney and chronic liver diseases have emerged in the HIV population. A considerable percentage of end-stage liver disease (ESLD) in HIV population is attributed to hepatitis C co-infection and reactivation, and a growing need for solid organ transplantation has emerged among those patients. On the other hand, several studies on liver transplantations of patients co-infected with human immunodeficiency virus (HIV) and hepatitis C virus (HCV) have shown discouraging results both in patient and graft survival rates. As a result, HIV-HCV co-infection has been considered a relative contraindication for liver transplantation. Thankfully, new drugs for HCV treatment have been discovered, acting direct on viral replication of HCV and they have changed the whole clinical course of HCV/HIV co-infected liver transplant recipients. Our case illustrates the long-term efficacy and safety of the new combination of Sofosbuvir/Ledipasvir in HCV/HIV co-infected liver transplant recipients.

\section{Introduction}

Widespread implementation of highly active antiretroviral therapy (HAART) has improved dramatically the life expectancy of HIV-infected patients [1] and with this increase in life expectancy, illnesses such as chronic kidney and chronic liver disease constitute a new challenge in HIV-infected patients [2]. Liver disease is now a leading cause of morbidity and mortality in the HIV-infected population [3]. A large proportion of liver diseases originates from HCV co-infection, because of shared routes of transmission. In the USA, $15-30 \%$ of HIV-infected patients are co-infected with HCV [4]. HIV infection has been associated with a more rapid progression of the HCV-related liver disease, and the risk of progression to end-stage liver disease (ESLD) in HIV-HCV co-infected patients is six-fold higher, in comparison with HCV mono-infected patients [5]. Additionally, HCV infection is leading cause for inferior outcomes after orthotopic live transplantation (OLT) in the patient with HIV/HCV co-infection [5]. Herein, we present a case report of a HIV-HCV co-infected patient who underwent OLT and had an HCV recurrence, which was treated successfully with a direct-acting antivirus regiment (DAAs) and has excellent two years survival to date.

\section{Case Presentations}

A 59-year-old Caucasian male presented in 2013 for liver transplant evaluation as the treatment for hepatocellular carcinoma (HCC), on the background of liver cirrhosis from HIV (on HAART) and HCV infection (without treatment). A routine hepatoma screening; via contrast triple phase CT-Scan demonstrated an arterially enhancing lesion within segment VIII of the liver measuring $1.8 \times 1.7 \times 1.8 \mathrm{~cm}$ and the lesion washed out on portal venous and delayed phases (OPTN V). His past medical history 
was significant for anxiety, depression, HIV and HCV infection (HCV genotype 1a) both diagnosed several years before. The patient had a positive smoking history which was stopped five years before and positive history for illegal drug abuse which was stopped ten years before. His past surgical history was significant for right nephrectomy and cholecystectomy after a gunshot wound. His family history was significant for coronary disease in both parents.

The patient underwent four sessions of trans-arterial chemoembolization (TACE) as a bridging maneuver to liver transplantation. After one and a half years from the initial diagnosis of the lesion, a suitable HCV negative deceased donor became available and the patient underwent OLT. At the time of transplantation, the patient had MELD score 34, WBC $6.8 \mathrm{~K} / \mathrm{MM}^{3}$, Hematocrit 39.2\%, Platelets: $62 \mathrm{~K} / \mathrm{MM}^{3}$, CD4 199cells/uL (CD4 percentage 43\%), Total Bilirubin $1.7 \mathrm{mg} / \mathrm{dL}$, Alanine Aminotransferase $79 \mathrm{U} / \mathrm{L}$, Aspartate Aminotransferase $87 \mathrm{U} / \mathrm{L}$ and AFP 10.4. The histological examination of the explant showed residual hepatocellular carcinoma confined to the liver, status post chemoembolization, moderate differentiated, with $90 \%$ tumor necrosis and without vascular or peri-neural invasion. The non-neoplastic liver was cirrhotic, stage 6/6, with chronic active hepatitis and macro-steatosis up to $25 \%$. The postoperative course was uneventful and the patient was discharged ten days later, with treatment for HIV (Raltegravir, Abacavir, Lamivudine and Maraviroc) and immunosuppression therapy with Steroids, Mycophenolate Mofetil and Tacrolimus. Two months after transplantation the patient came back with clinical and laboratory picture of acute cellular rejection, which was verified with biopsy and treated appropriately with steroids. After this episode of acute rejection, he was additionally treated three times with steroids. During the first post-operative months after the liver transplantation the patient had clinical and laboratory findings compatible with HCV recurrence (HCV PCR 8,571,687 International Units/mL) and it was proposed to start treatment for HCV infection, with a combination of Ribavirin, Ledipasvir and Sofosbuvir. He received 24 weeks of anti-HCV treatment and sustained virologic response (SVR) was achieved for both 12 and 24 weeks. One year after the completion of HCV treatment the patient had no detectable levels of HCV and no adverse effects or drug interactions have been observed. Preoperative and postoperative laboratory findings are cited in table 1 and the $\mathrm{HCV}$ viral load course is cited in figure 1.

\section{Discussion}

HCV-HIV co-infection is associated with exceedingly poor outcomes after OLT, with regard to both patient and graft survival rates; mainly due to a higher rate and worse phenotypes of rejection in that population [5]. The causative factors are primarily hepatitis $\mathrm{C}$ virus recurrence which leads to liver fibrosis/cirrhosis and HIV infection which accelerates the pathophysiologic process, through immunosuppression [6]. Therefore, in HIV-HCV co-infected liver transplant candidates there are two primary

\begin{tabular}{|c|c|c|c|c|c|c|c|c|c|c|}
\hline \multirow{2}{*}{ Dates } & \multirow{2}{*}{ AST } & \multirow{2}{*}{ ALT } & \multirow{2}{*}{ ALP } & \multirow{2}{*}{ T.BILI } & HCV RNA & \multirow{2}{*}{$\begin{array}{c}\text { HIV RNA } \\
\text { (Copies/mL) }\end{array}$} & CD4 & $\mathrm{Hb}$ & WBC & PLT \\
\hline & & & & & (IU/mL) & & count & $(\mathrm{gm} / \mathrm{dL})$ & (K/MM3) & (K/M M3) \\
\hline \multicolumn{11}{|c|}{ Preoperative Values } \\
\hline Oct 13 & 62 & 74 & 150 & 0.8 & 66,721 & N/A & $\mathrm{N} / \mathrm{A}$ & 12 & 3 & 96 \\
\hline Jan 14 & 147 & 179 & 204 & 1.2 & 601,785 & $<20$ & $\mathrm{~N} / \mathrm{A}$ & 12.8 & 3.5 & 91 \\
\hline May 14 & 22 & 20 & 119 & 0.6 & 13,293 & N/A & 29 & 10.9 & 2 & 109 \\
\hline \multicolumn{11}{|c|}{ Postoperative Values } \\
\hline Feb 15 & 1271 & 562 & 63 & 3.1 & 153,790 & N/A & 199 & 13.3 & 6.8 & 62 \\
\hline Mar 15 & 173 & 199 & 813 & 1.1 & $2,117,177$ & $<20$ & 135 & 11.7 & 2.6 & 146 \\
\hline May 15 & 358 & 330 & 327 & 4.6 & $8,571,687$ & $<20$ & 102 & 11.8 & 3 & 107 \\
\hline June 15 & 28 & 24 & 122 & 1 & 1,259 & $<20$ & N/A & 9.1 & 3.9 & 195 \\
\hline July 15 & 17 & 25 & 128 & 0.9 & $<15$ & N/A & N/A & 9.9 & 4.5 & 166 \\
\hline Aug 15 & 31 & 67 & 120 & 0.4 & $<15$ & $<20$ & 320 & 11.3 & 6.5 & 198 \\
\hline Feb 16 & 25 & 62 & 343 & 0.4 & $<15$ & N/A & 182 & 11.1 & 5.1 & 194 \\
\hline May 16 & 47 & 91 & 403 & 0.3 & $<15$ & N/A & N/A & 11.8 & 5.9 & 120 \\
\hline
\end{tabular}




\section{Hepatitis C Virus Quantitative Real Time PCR}

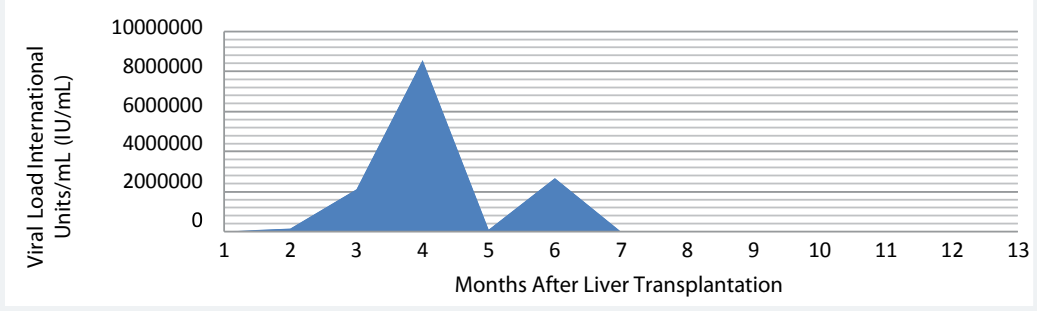

Figure 1: Patient's HCV viral load course.

treatment targets, HIV and HCV, both of which should be managed sufficiently and aggressively. Hepatitis C virus control is the key factor, as HIV has been controlled adequately with HAART, and it can be implemented either in pre-transplantation or in post-transplantation period. The main goal of the HCV treatment is the achievement of undetectable HCV-RNA levels for a long period after the treatment completion, which has been called sustained virologic response (SVR). Pearlman et al have defined sustained virologic response (SVR) as "aviremia" 24 weeks after completion of antiviral therapy for chronic hepatitis C virus (HCV) infection [7]. In the past, Pegylated interferon (PegIFN) and ribavirin (RBV) were the only available HCV treatment choices. However, both have shown very poor outcomes, with limited efficacy and poor tolerance [4]. Fortunately, during the last couple of years that dismal picture has changed dramatically mainly because discovery of DAAs. DAAs target specific proteins-enzymes of HCV life cycle and can be categorized into four main categories: NS5A inhibitors, NS3/4A protease inhibitors, NS5B polymerase inhibitors (nucleoside and non- nucleoside) and NS5A inhibitors [8]. A number of recent studies have reported exceptional outcomes in HCV mono-infected patients, with SVR 12 in $94 \%$ and SVR 24 in 99\% of treated population [9,10]. However, HCV treatment in HIV/ HCV co-infected liver transplant candidates and furthermore in HIV/HCV co-infected liver transplant recipients is intricate and demanding. Balancing immunosuppression with minimizing HCV recurrence and HIV infection is challenging and often counterintuitive. As observed in our case, the immunosuppression after liver transplantation in HIV-HCV co-infected patients can suppress the recipient's defenses to such a degree as to facilitate early HCV recurrence and can lead to liver transplant rejection. Possible underlying mechanisms are the inadequate immunosuppression due to drug interactions between ART medications and immunosuppressant, and cross-reactivity between memory T cells, with subsequent hepatocellular injury $[5,11,12]$. Treatment of rejection with steroids creates a vicious cycle, by suppressing the immune system and accelerating HCV recurrence. A key to breaking the vicious cycle of rejection is the new direct-acting antiviral drugs for HCV as demonstrated in this case with the combination therapy of Ribavirin, Ledipasvir and Sofosbuvir. This demonstrates that DAAs can be used safely to achieve long term and durable SVR after treatment of HCV recurrence in HIV-HCV co-infected liver transplanted patients, without serious drug interactions or adverse effects.

One of the major concerns in treating the HIV-HCV co-infected liver transplant recipients is the drug interactions between antiretroviral therapy, anti-HCV therapy and immunosuppressive therapy. The first approved HCV protease inhibitors (PIs), boceprevir and telavapir, are potent inhibitors of cytochrome P450 3A (CYP3A), demonstrating serious DDIs with HIV protease inhibitors and several non-nucleoside reverse transcriptase inhibitors (NNRTIs) [13]. It would be safer to avoid combination of those DAAs with efavirenz. Furthermore, by inhibiting the CYP3A4 there is a need for reduction of the dosages in calcineurin inhibitors (CNIs), such as tacrolimus/ cyclosporine and mammalian target of rapamycin inhibitors like sirolimus (MTORi) [13]. However, the combination of Sofosbuvir/Ledipasvir for HCV treatment has shown 
a safer drug interaction profile and the co-administration with Raltegravir, Abacavir, Lamivudine and Tacrolimus seems to be safe and without need for medication dose adjustment.

A limited number of studies have addressed the HCV treatment with DAAs, in $\mathrm{HCV} / \mathrm{HIV}$ co-infected liver transplant recipients. Grant et al. has described eight HCV/ HIV co-infected liver transplant recipients, six with genotype 1a, one with genotype $1 \mathrm{~b}$ and one with genotype $2 \mathrm{~b}$, who were treated with either Sofosbuvir/Ribavirin or Sofosbuvir-Simeprevir-Ribavirin [14]. Seven out of the total eight patients completed a 12 weeks therapy and all achieved SVR 12 without significant adverse drug interactions. Campos-Varela et al. has described two cases of HCV/HIV co-infected liver transplant recipients, both genotype $1 \mathrm{a}$, who were treated with DAAs and both achieved SVR 12 [15]. Alkhouri et al. have described a case of successful HCV treatment of a HIV liver transplant recipient with the combination of Telaprevir/Ribavirin/ Pegylated Interferon [16]. Future studies with larger populations are needed to evaluate the possibility of treating HCV/HIV liver transplant recipients with DAAs and their interactions with immunosuppressive and antiretroviral medications. The advent of DAA drugs has given the opportunity in HCV-HIV population to counter the deleterious effect of HCV on liver allografts after transplantation. In our opinion HIV$\mathrm{HCV}$ co-infection is no longer a contraindication for liver transplantation.

\section{References}

1. Palella FJ Jr, Delaney KM, Moorman AC, Loveless MO, Fuhrer J, et al. Declining morbidity and mortality among patients with advanced human immunodeficiency virus infection. HIV Outpatient Study Investigators. N Engl J Med. 1998; 338: 853-860. Ref.: https://goo.gl/NKoaxq

2. Smith CJ, Ryom L, Weber R, Morlat P, Pradier C, et al. Trends in underlying causes of death in people with HIV from 1999 to 2011 (D:A:D): a multicohort collaboration. Lancet. 2014; 384: 241-248. Ref.: https://goo.gl/RbEuFa

3. Bica I, McGovern B, Dhar R, Stone D, McGowan K, et al., Increasing mortality due to end-stage liver disease in patients with human immunodeficiency virus infection. Clin Infect Dis. 2001; 32: 492-497. Ref.: https://goo.gl/JCgaT2

4. Sulkowski MS. Hepatitis $C$ virus infection in HIV-infected patients. Curr HIV/AIDS Rep. 2004; 1: 128135. Ref.: https://goo.gl/UPnMpF

5. Kardashian AA, Price JC. Hepatitis $\mathrm{C}$ virus-HIV-coinfected patients and liver transplantation. Curr Opin Organ Transplant. 2015; 20:276-285. Ref.: https://goo.gl/BZ2dbo

6. de Vera ME, Dvorchik I, Tom K, Eghtesad B, Thai N, et al. Survival of liver transplant patients coinfected with HIV and HCV is adversely impacted by recurrent hepatitis C. Am J Transplant. 2006; 6: 29832993. Ref.: https://goo.gl/Zkvzq2

7. Pearlman $B L$, Traub N. Sustained virologic response to antiviral therapy for chronic hepatitis $\mathrm{C}$ virus infection: a cure and so much more. Clin Infect Dis. 2011; 52: 889-900. Ref.: https://goo.gl/jtRmH8

8. Poordad F, Dieterich D. Treating hepatitis C: current standard of care and emerging direct- acting antiviral agents. J Viral Hepat. 2012; 19: 449-464. Ref.: https://goo.gl/DxaFKS

9. Afdhal N, Reddy KR, Nelson DR, Lawitz E, Gordon SC, et al. Ledipasvir and sofosbuvir for previously treated HCV genotype 1 infection. N Engl J Med. 2014; 370: 1483-1493. Ref.: https://goo.gl/JEj6LZ

10. Afdhal N, Zeuzem S, Kwo P, Chojkier M, Gitlin N, et al. Ledipasvir and sofosbuvir for untreated HCV genotype 1 infection. N Engl J Med. 2014; 370: 1889-1898. Ref.: https://goo.gl/NFgYh2

11. Frassetto LA, Browne M, Cheng A, Wolfe AR, Ronald ME, et al. Immunosuppressant pharmacokinetics and dosing modifications in HIV-1 infected liver and kidney transplant recipients. Am J Transplant. 2007; 7: 2816-2820. Ref.: https://goo.gl/2GCzb6

12. Wu Z, Bensinger SJ, Zhang J, Chen C, Yuan X, et al. Homeostatic proliferation is a barrier to transplantation tolerance. Nat Med. 2004; 10: 87-92. Ref.: https://goo.gl/SvpVXw

13. Wilby KJ, Greanya ED, Ford JA, Yoshida EM, Partovi N, et al. A review of drug interactions with boceprevir and telaprevir: implications for HIV and transplant patients. Ann Hepatol. 2012; 11: 179185. Ref.: https://goo.gl/CsY2yS 
14. Grant JL, Hawkins C, Brooks H, Frank J Palella, Sean WP Koppe, et al. Successful sofosbuvir-based therapy in HIV/hepatitis $C$ virus coinfected liver transplant recipients with recurrent hepatitis $C$ virus infection. AIDS. 2016; 30: 93-98. Ref.: https://goo.gl/yYx5PH

15. Campos-Varela I, Stephanie S, Eliana Z Agudelo, Laurie C, Norah AT. Sofosbuvir, simeprevir, and ribavirin for the treatment of hepatitis $C$ virus recurrence in human immunodeficiency virus/ hepatitis C virus-coinfected liver transplant recipients. Liver Transpl. 2015; 21: 272-274. Ref.: https://goo.gl/Wz1Nwn

16. Alkhouri N, Singh G, Carey WD, Fung JJ, Zein NN, et al. The successful use of telaprevir to treat hepatitis $\mathrm{C}$ recurrence afterliver transplantation in an HIV co-infected patient. Transplantation. 2014; 97: 14-15. Ref.: https://goo.gl/s17rVev 